\title{
Malfunction of the masticatory system (Temperomandibular disorders TMD) and malocclusion:

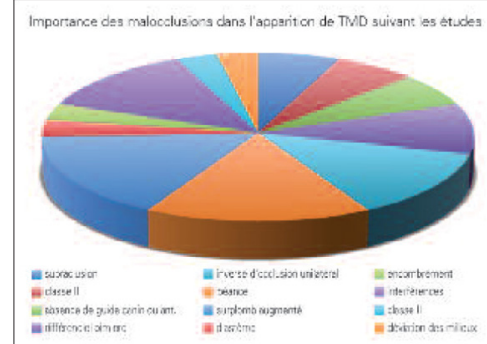 is there a relationship? A systematic revue of the literature from 2000 to 2009
}

\author{
Lorraine BELOTTE-LAUPIE, Milléwa SAYAGH, \\ Armelle MANIÈRE-EZVAN
}

\section{ABSTRACT}

Many hypotheses concerning the relationships that might exist between the disorders of masticatory system or temperomandibular disorders (TMD) and malocclusions have been advanced. But, in terms of evidence-based medicine, can we say that any of them have scientific merit?

The objective of our project was to analyze the existing international literature of the years 2000 to 2009 on the relationship between malocclusion and malfunction of the masticatory system.

Materials and method: we used MEDLINE to find all papers written in French or English that had an abstract referring to "Malocclusion" and "Temperomandibular joint disorders." We subjected them to a critical analysis using a reading record program that sorted the papers into types of study, descriptive or analytical, and the intensity of their methodology. Two of our authors calibrated this analysis and we compared their results.

Results: We examined 17 studies out of a panel of 171 articles. Nine out of the seventeen were transverse studies, six were longitudinal studies, one evaluated cases and a control group, and one was a cohort study. An ethical committee had approved of 9 of the studies and 9 out of the group had been randomized. From all seventeen we collected calibrations from observers and made statistical analyses of the data. 
Our conclusion was that there is a relationship between malocclusion and TMD but that it is a weak one.

The results of the different studies varied considerably as a factor of the type of malocclusion incriminated and the type of temperomandibular disorder reported. Our work indicated that a relationship between TMD and malocclusion cannot be established and the studies we reviewed had little scientific value primarily because of the heterogeneity of the samples and of the lack of clearly established definitions of the terms "malocclusion" and "disorder" of the masticatory system.

\section{KEYWORDS}

\section{Malocclusions,}

Malfunction of the masticatory system,

Systematic review of the literature,

TMD (Temperomandibular disorder).

\section{1 - INTRODUCTION}

In spite of many attempts to elucidate the supposed role that occlusion plays in the development of disorders of the masticatory system over the past thirty years, no one has yet produced any significant or clinical evidence that it is, in fact, an etiological factor even though for many years dentists accepted pronouncements that it was a principal agent in the disorder. There is today no consensus that malocclusions play a major, a minor, or any role at all in the development of disorders of the masticatory system (TMD), which are said to have a multifactorial etiology. For the supposed contribution of malocclusion, Mackie and Lyons ${ }^{16}$ cited the stress and psychological difficulties associated with it, noxious habits, systemic factors, and trauma. De Boever (cited by Landi ${ }^{13}$ ), in a systematic 2000 review of the literature reported that many occlusal factors were associated with disorders of the masticatory system, but that this association was not "strong." The goal of our work is to focus on studies carried out since 2000, to assess their protocols, to analyze their scientific value, and to determine if it is possible to draw any significant conclusions on the relationship that might exist between malocclusion and disorders of the masticatory system, in order to reduce uncertainty about the issue and help orthodontists gain a better understanding about our functioning in our daily practices. 


\section{2 - PROTOCOL OF THIS STUDY}

We propose to employ the four axes proposed by ANAES ${ }^{1}$ :

Identification of information,

Selection of articles to study,

Analysis of articles,

Synthesis.

\section{2 - 1 - Title}

Malocclusions and disorders of the masticatory system: is there a relationship?

\section{2 - 2 - Objective of the study}

In this work we propose to evaluate the quality of the methodology of published studies on the relationship between malocclusion and disorders of the masticatory system. In a second section, we examine these studies

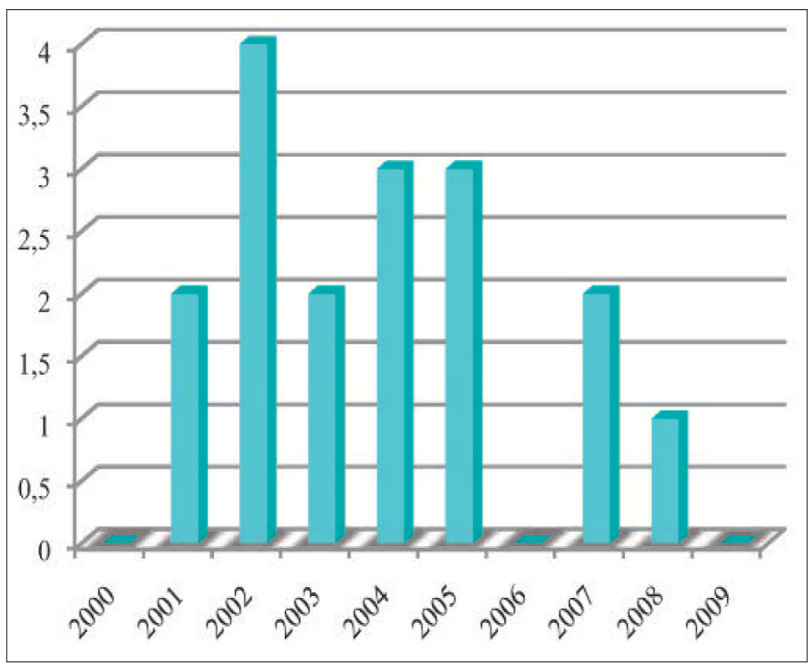

Figure 1

Number of studies published per year. with respect to their methodological and/or ethical criteria.

\section{2 - 3 - Methods and materials (or "identification of information) $6,12,15$}

\section{2-3-1 - Concept of the database}

- Definition of data base: a stock or bank of data that is a collection of digital information related to defined area of knowledge, organized and structured to facilitate location of details and access to them by means of devices that are usually electronic.

\section{- Criteria for the selection of articles}

We decided to choose journal articles published in English or French between 2000 and 2009 that included

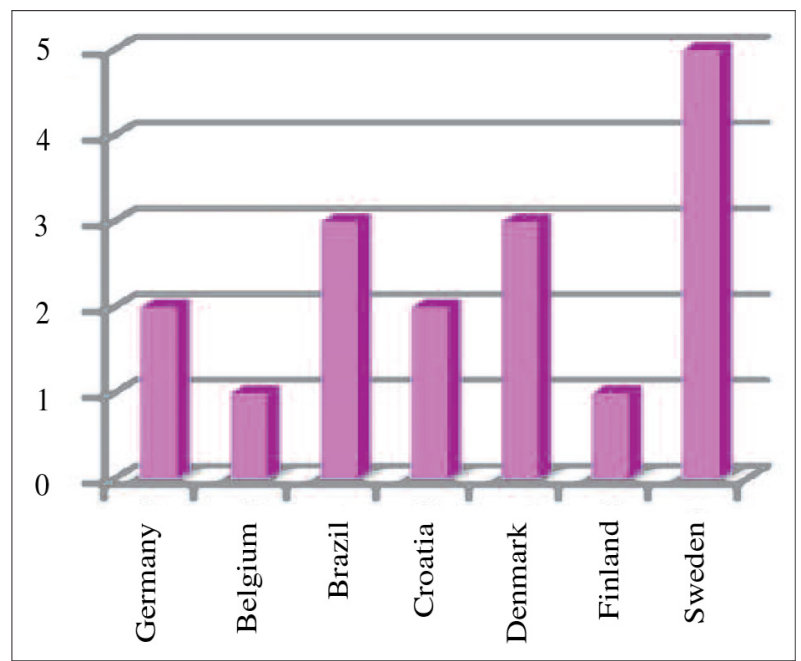

Figure 2

Number of studies per country. 
the key words "malocclusion" and "TMD" that had an abstract, and were not case reports or literature reviews.

\section{- Sources of information}

- The medical data bank, MED-LINE, for English language medical literature, Analysis and retrieved Systems Online, the most important digitalized, analytical bibliographical data base produced by United States National Institute of Health's National Library of Medicine. It references worldwide biomedical literature and is updated daily. But it does not actually provide complete world coverage because no database could possibly contain everything. So if Medline fails to produce results, researchers must look elsewhere.

We decided to choose journal articles published in English or French between 2000 and 2009 to evaluate the methodological quality of studies using data bases like Aidsline, Embase, Toxline and others to which we gained access through Pubmed (http: //wwwpubmed.org).

- The "grey" literature In ANAES (which has become HAS), "there are non-indexed official catalogues, conventional information circuits, abstracts from conventions, governmental documents, unpublished studies, reports and other unconventional documents, all of which make it an important source of information.

\section{- Accessing the database}

- We searched through PUBMED without using the MeSh the- saurus for the subject "Malocclusions and TMD, is there a relationship?" Accordingly we had to conduct our search with a combination of key words, "malocclusions" and "TMD" in a time frame of 2000 to 2009 . So we harvested two groups of articles, reflecting two sets of criteria applied in the same time period. Then we applied additional criteria, the first being presence of an abstract, inclusion of a tag which helped us to eliminate review articles, case reports, interviews, letters, and editorials. After having winnowed our crop by type, we then limited the harvest by language, English or French only.

- Results of the search

Our initial yield was consisted of 3,244 TMD articles and 6,350 malocclusion articles for the time segment of 2000 to 2009. Then we reduced the groups to articles that had an intersection of TMD and malocclusion, leaving a final list of 171 articles.

- List of articles obtained from MEDLINE:

In order to be certain our list contained only articles dealing with malocclusion and TMD we reviewed our list manually to remove those focused on orthognathic surgery, treatment with oral splints, and kinesitherapy, subjects we did not evaluate in our analysis.

- Two readers performed the triage in order to reduce the risk of individual error. After the first selection, 51 articles remained in our list.

- In a third weeding out process, we eliminated articles that: 
* were long-term studies with no recent results;

* articles that viewed malocclusion in terms of "occlusodontics" not orthodontics;

* articles whose sole goal was to establish a relationship between orthodontics and TMD;

* were literature reviews, not studies;

This left us with our group of 17 articles whose authors were:

- L. SONNESSEN, et al. (3 articles) 22,23,24,

- E. Carlson, et al. (1 article) $)^{3}$,

- R. Celic, et al. (2 articles $)^{4,5}$,

- B. Thilander, et al. $(1 \text { article })^{25,}$

- B. Mohlin, et al. $(1 \text { article })^{18,}$

- CM. Selaimen, et al. (1 article $)^{21,}$

- T. Magnusson, et al. $(1 \text { article })^{17}$.

- C. HIRSCH (1 article) ${ }^{11}$

- D. GeSCH, et al. (1 article) ${ }^{8}$,

- R. PAHKala, et al. $(1 \text { article })^{19}$,

- V. Tuerlings, et al. $(1 \text { article })^{26,}$

- A. Conti, et al. $(1 \text { article })^{7}$.

- T. HenRIKSON and Nilner $(1 \text { article })^{10,}$

- F. Godoy, A. Rosenblatt, J. Bezerra $(1 \text { article })^{9}$.

\section{2-3-2 - Critical analysis of the articles (our "methodology") 14,15}

\section{- General terms}

After the first two identification and selection phases of article selection we analyzed the articles in order to harvest information we could use in our synthetic document. This literature analysis constituted the initial stage of our preparation of medical and professional recommendations and references that would guarantee quality.
For this work we used a reading grid that we adapted from the "Critical Appraisal Worksheet" of the Centre for Clinical Epidemiology and Biostatistics of the University of Newcastle, New South Wales, Australia, which consists of eight evaluation criteria: the objective of the study, the type of protocol employed, the factors studied, the judgment criteria, the population source and subjects examined, the statistical analyses, the possible biases, the statistical analyses, and the author's conclusions (Guide of analysis of the literature and gradation of recommendations, ANAES 2001).

\section{- Evaluation of criteria}

Readers need landmarks that help them decode and sort information in an effective and standardized fashion so that they can more easily identify the strengths and the weaknesses of an article as well the inclusion of points that weaken an article's validity.

- The objective of our study

A critical reading gives us the necessary information to place an article in an appropriate rubric in order to clarify its objective that may be to elucidate: the history of the malady, the prognosis and development of the malady, the mode of utilization, performance, the benefits of a diagnostic test, the impact of therapy, and the determination of an etiology and causality, in terms of our study, malocclusion and TMD.

- The type of study and the quality of proofs it presents are etiological. According ANAES the most satisfactory protocols for judging causality are, in ranking order of the level of proof: random trials, cohort studies, and case studies with control groups. 
- The factors studied (here the criteria for evaluation of the malocclusion). The factor we studied was the intervention that was designed to cause favorable changes in the health problem, a malady or clinical condition.

The questions the reader should formulate:

- how were the factor, or factors, measured?

- were all the possibly pertinent factors taken into account?

- were the measurement methods applied equivalently to all subjects and all groups?

- what is the resultant factor or judgment criterion (here for evaluating TMD)?

- The judgment criterion is the event or situation supposed to have resulted from the influence of the factor being studied, such as death, disease, handicap, discomfort, and dissatisfaction. The reader should also be attentive to the precision with the definition of the resultant factor is framed as well as the measurement method so that a final synthesis of the results can be made.

- Population and sample studied

The reference population or the population being studied is the group for which the results of the study can be applied if they are valid. The sample is a sub-group of the population studied, sometimes selected randomly, sometimes not, that reflects the characteristics of the population as a whole when it is not possible, for practical reasons, to evaluate that entire population. The subjects in the sample may be segregated into groups.
Questions to ask:

- was the selection correct?

- was it randomized? (dividing the sample or the population into two or more comparable groups with a method that is based on chance);

- were the groups differentiated by characteristics other than the factors being studied?

- what proportion of the subjects was available to follow-ups? According to ANAES ${ }^{1}$, the characteristics of populations exposed and not exposed, sick and control, that determined the criteria for inclusion or exclusion, must be known, especially if the risk factors and malady being studied can be related to these characteristics.

- Possible bias

Bias occurs in subject selections that are not randomized.

- The results

The reader must know if the results derive from chance or statistically represent an action of the factor under examination. Variables help to answer this question. The value $p$ stands for the probability, calculated by the test statistic constructed from the collected data, of chance having caused differences equal to or greater than the difference that was observed. A result is called statistically significant if it is unlikely to have occurred by chance. Statisticians usually employ significance levels of $1 \%$ or $5 \%$, which, in means that there are one chance or five chances out of 100 that the difference observed was due to chance and not the factor observed. If, then, the significance level chosen is $5 \%$, which is usually the case; that 
means that $p<0,05 \%$ signifying that the effect is statistically significant, that is, due to something other than chance.

- Conception of the analysis grid

The conception of our reader's analysis grid is based on the Landrivon $^{14}$ grid, which an adaptation of the "Critical Appraisal Worksheet."

\section{3 - RÉSULTS OF THE CRITICAL READING}

The 17 studies that survived our weeding out process constituted our sample.

- Type of study: nine of the seventeen were transverse studies, six were longitudinal, one was a case study with a control group, and one was a cohort study.

- Ethical committee: An ethical committee approved nine out of the seventeen studies in our sample.

- Randomization: Nine studies were randomized.

- Calibration: Observers calibrated fourteen of the studies.

- Statistics: All seventeen of the articles in our group were statistical studies.

- Synthesis of the results: Godoy, et al. ${ }^{9}$ concluded that patients whose teeth were maloccluded were at no greater risk for developing TMD than persons who did not have malocclusions. Tuerling ${ }^{26}$ came to the same conclusion that there was no correlation between TMD and malocclusion in infants, who have a great capacity for adaptation.
The reading grid of an article on causality helps the reader answer these questions: is there an association between the risk factor and the malady, is this relationship strong, can the risk factor have caused the effect?

We prepared a record sheet for each article in our study.

According to Sonnessen, et al. ${ }^{24}$, overbite, and especially lingual inclination of the maxillary incisors, are risk factors for TMD and Pahkala ${ }^{19}$ asserted that pronounced overjet and anterior open bite in children both increased the risk for developing muscular sensitivity. But Hirsch ${ }^{11}$ concluded that there was no relationship between overbite, even in extreme cases, and the objective TMD symptoms. On the other hand Conti's study $^{7}$ came to the conclusion that there was a statistically significant relationship between the absence of anterior guidance and the presence of as well as the severity of TMD.

Selaimen ${ }^{21}$ found that Class II malocclusions and lack of canine guidance increase the prevalence of TMD. Celic ${ }^{4}$ work concluded that people with Class I malocclusions with an overjet greater $5 \mathrm{~mm}$, Class II division 2 malocclusions, anterior cross bites, midline deviations, and non-working contacts in working mandibular movements have increased risk of TMD but that the relationship is weak. Thilander's study reported a significant association between TMD and posterior cross bites, Class III malocclusions, and severe overjets. 
Magnusson, et al. ${ }^{17}$ found a significant correlation between TMD and a forced lateral occlusion, a vertical or sagittal differential between centric relation occlusion and maximal intercuspation occlusion, and unilateral cross bite, but no relationship between over bite and occlusal interferences with TMD. Landi ${ }^{13}$ found that only a differential greater than $2 \mathrm{~mm}$ between centric and maximum intercuspation occlusion increased the risk of facial muscular pain.

\section{4 - DISCUSSION}

Having collected such a large descriptive panoply of factors studied and results presented, we decided to concentrate exclusively on malocclusions and TMD problems and the precise manner in which the authors had defined and measured them. That is why we searched in each study for conclusions that reported the existence or absence of a relationship between malocclusion and TMD.

Our sample, derived exclusively from a sector of the literature delimited by the years 2000 to 2009, was comprised of studies whose results were totally divergent!

This could be explained by the lack of harmony in the form and parameters of the studies, both of which contributed to the bias we found in them.

\section{$4-1$ - The form of the studies}

- The sample population: there was a lack of harmony in the
Sonnesen ${ }^{23}$ found the most frequently occurring sign of TMD in cross bite cases, associated with a severe transverse discrepancy and an acute gonial angle, was sensitivity of the superficial masseter and anterior temporal muscles. And Gesch ${ }^{8}$ described a relationship between TMD problems and diastemas. Finally, Calrson ${ }^{3}$ asserted that tooth abrasion and overbite during childhood were significant predictors of an increase in clinical malfunction.

selection of the study samples in size, some working with dozens of cases, others with thousands. The age groups varied widely, ranging from children to adolescents, adults, and seniors. The origins of the groups were different, some were school children, some came from the framework of a previous study. Some were predetermined, grouping males and females separately; others were random from the beginning of the study. So we cannot be sure that the results we examined were representative of the general population.

- The follow-up: the signs and symptoms of TMD fluctuate over time so some of the studies were too limited in length of observation. Moreover, in transverse studies, delays in the appearance of problems were inconsistent or non-existent. 


\section{4 - 2 - The parameters (or factors studied)}

- The malocclusions: the authors of the papers in our study did not agree in their definition of malocclusion. Some focused their research on "functional malocclusions", or interferences, while others included "morphological malocclusions. Different authors assessed malocclusions in different ways making it impossible for an eventual correlation between malocclusion and TMD to emerge from their work. Furthermore, words have different meanings in different countries, for example an "occlusal interference" in the Nordic countries corresponds in French to "une prématurité occlusale" or occlusal prematurity. Furthermore, some authors are imprecise, failing to mention whether a "Class II malocclusion" was dental or skeletal or whether a cross bite was unilateral or bilateral.

- TMD: the method of assessing TMD in clinical examinations varies as well. Some clinicians listen for audible clicks in the tempero-mandibular joint while others use a stethoscope to detect them. Others palpate the joint searching for irregularities.

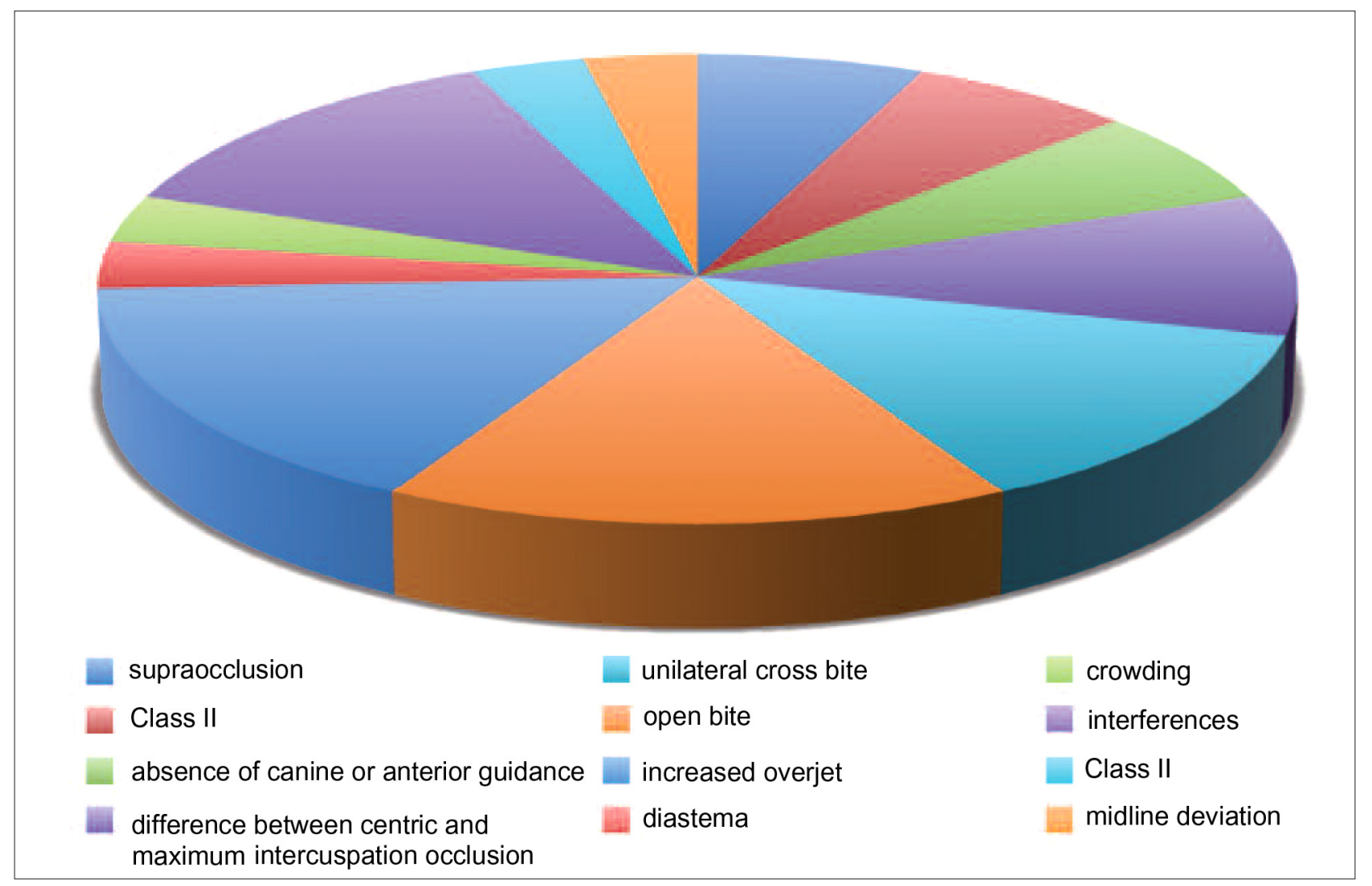

Figure 3

Malocclusions and TMD in the sample studied. 
And assessing muscular sensitivity by means of a Helkimo or McNeill index remains subjec-

\section{5 - CONCLUSION}

Our study affirms that there is no consensus about the relationship between TMD and malocclusion. Studies published on the subject have little scientific value, partly because of the bias in sample selection and partly because of the disparity in the definition of the basic terms, malocclusion and Tempero-Mandibular Disorders or TMD.

In order for researchers to undertake TMD projects that have real scientific merit they must join in an international agreement on the definition of TMD and devise an accepted diagnostically reliable classification for it. It seems to us indispensable for future research that we have a uniform conception of the nature of the problem.

In 2010 Cairns, List, Michelotti, Ohrbach and Svensson ${ }^{2}$ proposed in the Journal of Oral Rehabilitation a number of recommendations:

- The necessity of defining TMD.

- The necessity of defining acceptable occlusion. Today, in effect, any occlusion that is not ideal is considered to be a malocclusion. It would be useful to create subgroups of malocclusion and of TMD.

- The necessity of incorporating the principles of evidence based medicine into rigorous protocols for future studies. We cannot yet, it is true, demonstrate and link between TMD and malocclu- tive, all of which make calibration of TMD difficult. sion but there is no scientific proof that demonstrates the $a b$ sence of such a link. Accordingly, we must keep the multi-factorial etiology of TMD carefully in mind and remember that the appearance of temperomandibular joint symptoms also depends on the individual patient's capacity to adapt. In this view, orthodontics does not appear to be, in itself the prime factor in the prevention or the treatment of TMD. Nevertheless, orthodontists should strive to achieve treatment results whose occlusions respond to rigorous occluso-functional criteria. Successful orthodontic treatment does not consist solely of well-aligned teeth and a Class I occlusion. In order to assure that the teeth of our patients are able to withstand the extra constraints imposed open them by modern life, they must be as well organized as possible to adapt successfully to the demands of the 21 st century.

NdIR - the tables relating to the research that was used for the preparation of this study: Table /- file of publication analysis; Table II - list of publications of the sample, classified in decreasing chronological order; Table III - synthesis of the results by publication that can be consulted for more detail at the Revue d'ODF site : www. revue-odf.org. 


\section{REFERENCES}

1. ANAES: Guide d'analyse de la literature et gradation des recommandations, 2000. http://www.anaes.fr

2. Cairns B, List T, Michelotti A, Ohrbach R, Svenson P. JOR-CORE recommandations on rehabilitation of tempo- romandibular disorders. J Oral Rehabilit 2010;37(6):481-9.

3. Carlsson GE, Egermark I, Magnusson T. Predictors of signs and symptoms of temporomandibular disorders: a 20-years follow-up study from childhood to adulthood. Acta Odoltol Scand 2002;60(3): 180-5.

4. Celic R, Jerolimov V, Panduric J. A study of the influence of occlusal factors and parafunctional habits on the prevalence of signs and symptoms of TMD. Int $\mathrm{J}$ Prosthodont 2002;15(1):43-8.

5. Celic R, Jerolimov V. Association of horizontal and vertical overlap with prevalence of temporomandibular disor- ders. J Oral Rehabil 2002;29(6):588-93.

6. Chemin J, Houllier C. Malocclusions et dysfonctionnements de l'appareil manducateur : existe-t-il une association ? (revue de la literature) Thèse Chir Dent. Rennes : U Rennes I, 2007.

7. Conti A, Freitas M, Conti $P$, Henriques J, Janson G. Relationship between signs and symtoms of temporomandi- bular disorders and orthodontic treatment: a crosssectional study. Angle Orthod 2003;73(4): 411-7.

8. Gesch D, Bernhardt O, Mack F, John U, Kocher T, Alte D. Association of malocclusion and functional occlusion with subjective symtoms of TMD in adults: results of the Study of health in Pomeria (SHIP). Angle Orthod 2005;75(2):183-90.

9. Godoy F, Rosenblatt A, Godoy-Bezerra J. Temporomandibular disorders and associated factors in Brazilian tee- nagers: a cross sectional-study. Int J Prosthodont 2007;20(6):599-604.

10. Henrikson T, Nilner M. Temporomandibular disorders, occlusion ant orthodontic treatment. J Orthod 2003;30(2):129-37.

11. Hirsch C, John MT, Drangsholt MT, Mancl LA. Relationship between overbite/overjet and clicking or crepitus of tempromandibular joint. J Orofac Pain 2005;19(3):218-25.

12. Huguier M. Apprendre la lecture critique d'un article médical. Paris : Elsevier, 2003.

13. Landi N, Manfredini D, Tognini F, Romagnoli M, Bosco M. Quantification of the relative risk of multiple occlusal variables for muscle disorders of stomatognathic system. J Prosthet Dent 2004; 92(2):190-5.

14. Landrivon G. Méthode globale de lecture critique d'articles médicaux à l'usage des étudiants et du praticien. Paris : Frison-Roche, 2000.

15. Lorette G, Grenier B. La lecture d'articles médicaux. Rueil-Malmaison : Doin, 2002.

16. Mackie A, Lyons K. The role of occlusion in temporomandibular disorders- a review of litterature. New Zealand Dental Journal 2008;104(1):54-9.

17. Magnusson T, Egermark I, Carlsson GE. A prospective investigation over two decades on signs and symptoms of temporomandibular disorders and associated variables. A final summary. Acta Odonl Scand 2005;63(2):99-109.

18. Mohlin BO, Derweduwen K, Pilley R, Kingdon A, Shaw WC, Kenealy P. Malocclusion and tempromandibular disorder: a comparaison of adolescents with moderate to severe dysfunction with those without signs and symptoms of temporomandibular disorders and their further development to 30 years of age. Angle Orthod 2004;74(3):319-27.

19. Pahkala R, Qvarnström M. Can temporomandibular dysfunction signs be predicted by early morphological or functional variables? Eur J Orthod 2004;26(4):367-73.

20. Plouviez PH. Traitement orthodontique et dysfonctions de l'articulation temporomandibulaire, une relation existe- t-elle ? Mémoire de CECSMO Toulouse : U P. Sabatier, 2006. 
21. Selaimen CM, Jeronymo JC, Brilhante DP, Lima EM, Grossi PK, Grossi ML. Occlusal risk factors for temporo- mandibular disorders. Angle Orthod 2007;77(3):471-7.

22. Sonnesen L, Bakke M, Solow B. Temporomandibular disorders in relation to craniofacial dimensions, head pos- ture and bite force in children selected for orthodontic treatment. Eur J Orthod 2001; 23(2):179-92.

23. Sonnesen L, Bakke M, Solow B. Bite force in pre-orthodontic children with unilateral cross-bite. Eur J Orthod 2001;23(6):741-9.

24. Sonnesen L, Svensson P. Temporomandibular disorders and psychological status in adult patients with a deep bite. Eur J Orthod 2008;30(6):621-9.

25. Thilander B, Rubio G, Pena L, de Mauorga C. Prevalence of temporomandibular dysfunction and its association with malocclusion in children and adolescents : an epidemiologic study related to specified stages of dental development. Angle Orthod 2002;72(2):146-54.

26. Tuerlings $\mathrm{V}$, Limme $\mathrm{M}$. The prevalence of temporomandibular joint dysfunction in the mixed dentition. Eur J Orthod 2004;26(3):311-2027. 